\title{
Index
}

\section{Modern Authors}

Adriaensen, L. $56 \mathrm{n}$

Aerschot, S. 3in

Anderson, L. 75, 75n

Axters, S. 31n, 37n

Baar, M. de 2, 16n, 17n, 36n, 37n, 56n, 67n, 7in, 72n, 73n, 74n, 76n, 79n, 286, 287

Baers, J. 241n

Bilinkoff, J. 17n, 18, 18n, 24n, 29n

Bill-Mrziglod, M. 3on

Bloch, M. $27 \mathrm{nn}$

Blommestijn, H. 11n, 12n, 39n

Boaga, E. 39, 54, 266

Bos, A. $2,15 \mathrm{n}, 139 \mathrm{n}, 243 \mathrm{n}, 287$

Bouchereaux, S. M. 39n

Bronselaer, M. 26n, 59n

Burke, P. 17n, 27n, 37n, 72, 72n

Burschel, P. 23n, 3on, 4on

Ceyssens, L. 27n, 43, 44, 44n, 58n, 96n, 99n, 105n, 106n, 112n

Chalmers, J. 2, $121 n$

Chevallier, M. 68n

Cloet, M. 31n, 32n, 4on

Conrad, A. $32 n$

Deblaere, A. 1, 11n, 13n, 15, 15n, 23n, 24n, 53, 53n, 54n, 56n, 58n, 71n, 72, 72n, 73, 73n, $78 \mathrm{n}, 83,83 \mathrm{n}, 92 \mathrm{n}, 98 \mathrm{n}, 104 \mathrm{n}, 241 \mathrm{n}, 252 \mathrm{n}$, 283n, 286

Denzinger, H. 43n

Dreiskämper, P. 243n

Dudon, P. 105n, 106n

Fohrer, G. $267 \mathrm{n}$

Frijhoff, W. 29, 29n, 30n, 27in

Grosso, G. 2, 7n, 92n, 113n, 121n, 252n, 284

Haeghen, F. van der $\quad 112 \mathrm{n}$

Harline, C. $32 \mathrm{n}$

Hense, E. 1, 2, 17n, 36n, 37n, 73n, 84n, 87, 127n, 129n, 235n, 243n, 287
Herpoel, S. 73, 73n

Hoffer, P. $45^{\mathrm{n}}$

Hofman, R. 86, 86n, 92n

Hoornaert, P. 1on

Hoppenbrouwers, V. 23n, 35n, 41n, 54, 54n, 55n, 88, 88n, 89, 90, 99n

Houtman-Desmedt, H. $24 \mathrm{n}$

Janssens, P. $\quad 25^{2 n}$

Jedin, H. $29 n$

Kavanaugh, K. 253n

Klueting, E. 84n, 87

Kotschner, J. 275n, 276n

Lehmann, H. 7n, 22n

Lesaffer, R. $\quad 127 \mathrm{n}$

Lima, G. R. $41 \mathrm{n}$

Lottin, A. 31n, 69n

Maland, D. $246 \mathrm{n}$

Malena, A. 104n, 105n, 110n

Marnef, G. 25n, 29n

Martinez Carretero, I. $54 \mathrm{n}$

Meijer, M. 74, 75n

Meerbeeck, M. van 2, 11n, 13n, 26n, 33n, 42n, 54n, 7in, 284

Meulemeester, M. 36n

Meeuwsen, V. 1, 2, 16n, 26n, 27n, 76n, 86, 86n, 92n, 139n, 254n, 266n, 269n, 285

Melchior a Sancta Maria $55 \mathrm{n}$

Merlier, J.R.A. $68 \mathrm{n}, 69 \mathrm{n}, 7 \mathrm{on}, 73 \mathrm{n}, 78 \mathrm{n}, 111 \mathrm{n}$, 241n, 242n, 254n, 255n, 256n

Mommaers, P. 13n, 16n, 3on

Monteiro, M. 18n, 30n, 32, 32n, 33n, 34n, 7on

Monticone, A. 96n, 106n

Motta Navarro, T. 54n

Ockeley, J. 44n

Pacho, E. $104 \mathrm{n}$

Panzer, S. 23n, 31n, 38n, 39n, 40n, 54n

Persoons, E. $56 \mathrm{n}, 58 \mathrm{n}$

Philippen, L.J.M. 31n, 32n, 36n

Plattig, M. 38n, 39n 
Porteman, K. 13n, 30n, 74, 74n

Possanzini, S. 54n, 88, 88n, 283n

Put, E. 3in

Quaghebeur, T. $36 \mathrm{n}$

Ranft, P. 7on

Rapley, E. 3on

Reinhard, W. $23 \mathrm{n}$

Rodriguez, O. 253n

Roeck, B. $23 \mathrm{n}$

Roegiers, J. 43n

Rooms, E. 24n, 25n, 135n, 243n, 244n

Roosbroeck, R. $24 \mathrm{n}$

Sainsaulieu, J. 37n, 55n

Schulz, S. 34n

Smet, J. 28n, 33n, 36n, 38n, 39n, 4on

Smits-Veldt, M.B. $74 \mathrm{n}$

Sonnino, P. $25 \mathrm{n}$

Spiertz, M.G. $42 \mathrm{n}$

Staring, A. $\quad 7,33 n, 53,53 n, 58 n, 111,111 n$

Steggink, O. 23n

Strasser, U. 3on

Trepp, A. Ch. 7n, 22n

Triest, M. 31n, 32n

Uyttenhove, L. 13n

Valabek, R. $54 \mathrm{n}$

Vate, E. van de $1,2,15 n, 16 n, 18 n, 27 n, 30 n$, 36n, 42n, 44n, 67, 85n, 89n, 121n, 169n, 253n, 283, 284, 286

Velde, J. F. $44 \mathrm{n}$

Venard, M. 3in

Vroede, M. de 32n, 70, 7on

Waaijman, K. 266, 266n, 267n, 269n, 27on, 272n, 275n, 276n

Watt, D. $79 n$

Weber, A. $36 \mathrm{n}, 75,75 \mathrm{n}$

Wessels, G. 4in, gon

Wijnhoven, D. $55^{\mathrm{n}}$

Wilson, P. 22n

Zoegevon Manteuffel, K. $\quad$ 275n

\section{Names and Personalities}

Acarie, Berbe $39 n$

Albert of Austria 24, 31

Albizzi, Francesco $\quad 96 n, 106,106 n$

Albroex, Jan 56

Anna of St. Bartholomew 35n, 273, 274, 275, $275 n$

Arentsz, Pieter 68

Augustine 43n, 69, 74, 246, 286

Ballaert, Jan van, see Michael of St. Augustine Beaucousin, Richard 39n

Begga 36n, 58n, 59n

Behourt, Peter 38, 39

Bellarmine, Robert 37,37n

Bell'Uomo, Gottardo 105

Benedict of Canfield 9, 71

Berghes, Alphons de 44, 44n

Bernard of Clairvaux $\quad 45,45 n$

Berulle, Pierre de 39n

Blanzy, Thomas 39n

Bois, Nicolaus du $112 n$

Bona, Giovanni $\quad 27,45,45$ n

Bonaventura 107, 107n

Boonen, Jacob 31, 43n

Borromeo, Carlo 29, 44n

Bossuet, Jaques-Bénigne 27

Bourignon, Antoinette $\quad 2,56 \mathrm{n}, 67,67 \mathrm{n}, 68$, 68n, 69, 69n, 70, 7on, 71, 72, 73, 74, 75, 76, $76 \mathrm{n}, 77,77 \mathrm{n}, 78,286,287,288$

Casanate, Girolamo $\quad$ 105, 105n, 107n

Carmelite father, first Director of Maria Petyt 9, $71,255 \mathrm{n}$

Catharine of Genoa $15 \mathrm{n}$

Catharine of Siena 46

Charles II (Spanish King) 44n, 113, 114, 114n, 128, 129, 129n, 152, 153, 170, 171, 171n, 182, 183, 183n, 212, 213, 216, 217, 247, 263, 264, 273, 285

Charpentier, Louis 38

Clovis 28

Coriache, Aimé $\quad 73,76$

Cort, Christian de 56, 72, 76, 77

Count of Mons 166,167 
Count of Monterrey, see Mendez de Haro, Juan Domingo

Damman, Petrus 112n

Daniel of the Virgin Mary 10, 33, 33n, 34n, 36 , 36n, 40n, 41n, 55, 55n, 273, 273n, 275n, $276 \mathrm{n}$

d'Hondt, Livinus 40

Diepenbeeck, Abraham van 275, 276

Elector of Brandenburg, see Frederick William

Elijah 2, 38, 266, 273, 274, 275, 276, 278, 279, $280,287,288$

Emperor, see Leopold I

Engrand, Françoise $\quad 58,58 \mathrm{n}$

Euphrasia 11, 11n, 57

Euphrosyna 11, 11n, 57

Favoriti, Agostino 106n

Ferdinand of Austria 24

Francis de Sales 29, 71

Francis Xavier $18,18 \mathrm{n}$

Frederick William (Elector of Brandenburg) 131n, $221 n$

French King, see Louis XIV

Gabriel of the Annunciation $\quad 35,35 \mathrm{n}$

Geldolphus, Iosephus 36n

Gertude of Helfta 46, 206, 207

Gillemans, Ignace $45 \mathrm{n}, 76$

Gorcum, Ian van 37n

Gores, Anna 46

Graet, Maximiliaan 26

Hadewijch 77

Hauchin, Johannes 31, 32

Herman of St. Norbert $36,36 \mathrm{n}$

Hooghe, Martin de 40, 40n

Ignatius of Loyola 29, 241

Innocent XI 44n, 106

Isabella of Spain 24, 31, 4on

Jacob of St. Antony 90

James of the Passion 41n, 42n, 55, 112n

Jansen, Cornelius $\quad$ 42, 43n, 246

John of Austria 152, 153, 153n, 16o, 161
John of St. Samson $\quad 28,28 n, 39,39 n$

John of the Cross 13, 71, 77

Kerckhove, Jan Vanden 68

Léon de St. Jean 28

Leopold I (Emperor) 35n, 131n, 152, 153, 153n, $160,161,214,215,216,217,221 n, 248,276$

Liebeke, Anna van 55

Linden, Maria van der $36 \mathrm{n}$

Lord Lucas 166, 167

Louis IX 28, 28n, 163n, 164, 165, 212, 213, 213n, 214, 215, 230, 231, 271

Louis XIII 24, 28

Louis XIV (French King) 1, 2, 25, 27, 28, 35n, 76, 76n, 94, 94n, 95, 95n, 96, 96n, 113, 114, $114 \mathrm{n}, 120,121,122,123,123 \mathrm{n}, 124,125,126,127$, 127n, 128, 129, 130, 131, 134, 135, 136, 137, 138, $140,142,143,144,145,146,147,148,149,150$, 151, 154, 155, 160, 161, 162, 163, 163n, 168, 169, $170,171,172,173,173 n, 174,175,176,177,178$, 179, 182, 183, 183n, 184, 185, 186, 187, 187n, 188, 189, 190, 191, 192, 193, 194, 195, 196, 197, 198, 199, 200, 201, 202, 203, 204, 205, 212, 213, 214, 215, 216, 217, 218, 219, 220, 221, 222, 223, 224, 225, 226, 227, 228, 229, 230, 231, 232, 233, 234, 235, 236, 237, 238, 239, 240, 243, 244, 245, 245n, 246, 247, 248, 249, 250, 252, 256, 257, 258, 259, 260, 261, 262, 263, $264,267,269,270,272,273,275,276,279$, 285

Lutgarde of Aywiers $129 n$

Maracci, Louis 106, 106n, 108

Marcus à Nativitate ion

Maria de Medici 28

Mariana of Austria (Spanish Queen Consort) 143, 143n, 144, 145, 170, 171, 171n

Maria Teresa (Spanish Princess) 25, 243

Marie Bon de l'Incarnation 110

Marius of St. James $\quad 112 n$

Mark of the Resurrection 90

Marshal Luxembourg, see MontmorencyBouteville, François Henri de

Mendez de Haro, Juan Domingo (Count of Monterrey) 27n, 150, 151, 151n, 202, 203, 203n, 217n, 248

Menghini, Tommaso 110 
Michael of St. Augustine (Jan van Ballaert) 2, 8, 9n, 10, 10n, 11, 12, 12n, 13, 14n, 15n, 17n, $23,27,27 \mathrm{n}, 28,35,35 \mathrm{n}, 41,41 \mathrm{n}, 42,42 \mathrm{n}, 43 \mathrm{n}$, 44, 44n, 54, 54n, 56n, 57, 58, 58n, 68, 71, 72, $76,78,83,86,86 \mathrm{n}, 87,88,89$, 9o, 9on, 92, 92n, 93, 93n, 94, 94n, 95, 96, 96n, 97, 98, 98n, 99, 99n, 100, 100n, 101, 101n, 102, 102n, 103, 103n, 104, 109, 110, 111, 111n, 112, 112n, 113, 113n, 116, 119, 169n, 171n, 173n, 240, 241, 242, 242n, 244, 248, 249, 250, 266, 266n, 268, $279,282,283,284,285,287$

Molinos, Miguel de 105

Monsignani, Eliseo $35 \mathrm{n}$

Monsignani, Angelo 46

Montmorency-Bouteville, François Henri de (Marshal Luxembourg) 123n

Neri, Philip 29

Noels, Pierre 76

Orsaghen, Catharina van $\quad 11,58,58 n, 59$

Peeters, George 55, 55n

Pelagius $\quad 76$

Petyt, Maria passim

Philip II 24

Philip IV 25

Philip of the Visitation $34 \mathrm{n}$

Pius V 30

Poelman, Adrianus 112n

Poiret, Pierre 68,68n

Potel, François 4on

Prince-Bishopric of Münster 243

Prince of Orange, see William III

Puttemans, Anna 36

Richelieu 24, 28

Rieuwertsz, Jan 68

Ruusbroec 77,39n

Sanderus, Antonius 53, 53n
Sebastian of St. Paul 89

Segneri, Paolo 105

Seraphinus of Jesus and Mary 12n, 44, 54, 89, 9o, 9on, 93, 93n, 94n, 96, 96n, 97, 99, 99n, 100n, 101, 101n, 102, 102n, 103n, 106n, 111, $111 n$ Serracino Inglott, Pius 83

Silvio, Henry 38, 39

Sister Hannes 59

Sister Maria $\quad$ 98, 99, 99n, 100, 10on, 101, 102, 102n, 103, 103n

Sister van der Poorten 46

Sixtus IV $35^{\mathrm{n}}$

Soetems, Elisabeth van 36

Soreth, John 34, 41, 41n

Spanish King, see Charles II

Spanish Princess, see Maria Teresa

Spanish Queen Consort, see Mariana of Austria

Spoelberch, Willem $252 \mathrm{n}$

Stracci, Theodore $33,33 n, 40,40 n$

Tanara, Sebastiaan Antonius $\quad$ 101, 101n, 102, 102n

Teresa of Avila 2, 17, 18, 23, 29, 35, 36, 36n, 37, 37n, 38, 39, 39n, 41, 46, 71, 72, 73, 73n, 74, 75, $78,107,107 \mathrm{n}, 108,252,252 \mathrm{n}, 254 \mathrm{n}, 255,256$, 257, 258, 259, 261, 262, 286, 287, 288

Thibault, Philip 38, 39, 39n

Thomas à Kempis 9, 71

Timothy of the Presentation 41n, 58n, 62n

Triest, Antoon 31, 4on

Villiers, Cosmas de 90, 113n

Vincentius of the Nativity 112n

Widenfeldt, Adam $\quad 45 \mathrm{n}$

Wijmen, Leo van 83

William III (Prince of Orange) 135n, 141n, 163n, 244 